\title{
Efficient Program Power Behavior Characterization
}

\author{
Chunling Hu Daniel A. Jiménez Ulrich Kremer \\ Department of Computer Science \\ \{chunling, djimenez, uli\}@cs.rutgers.edu \\ Rutgers University, Piscataway, NJ 08854
}

\begin{abstract}
Fine-grained program power behavior is useful in both evaluating power optimizations and observing power optimization opportunities. Detailed power simulation is time consuming and often inaccurate. Physical power measurement is faster and objective. However, fine-grained measurement generates enormous amounts of data in which locating important features is difficult, while coarse-grained measurement sacrifices important detail.

We present a program power behavior characterization infrastructure that identifies program phases, selects a representative interval of execution for each phase, and instruments the program to enable precise power measurement of these intervals to get their time-dependent power behavior. We show that the representative intervals accurately model the fine-grained time-dependent behavior of the program. They also accurately estimate the total energy of a program. Our compiler infrastructure allows for easy mapping between a measurement result and its corresponding source code. We improve the accuracy of our technique over previous work by using edge vectors, i.e., counts of traversals of control-flow edges, instead of basic block vectors, as well as incorporating event counters into our phase classification.

We validate our infrastructure through the physical power measurement of 10 SPEC CPU 2000 integer benchmarks on an Intel Pentium 4 system. We show that using edge vectors reduces the error of estimating total program energy by $35 \%$ over using basic block vectors, and using edge vectors plus event counters reduces the error of estimating the fine-grained time-dependent power profile by $22 \%$ over using basic block vectors.
\end{abstract}

\section{Introduction}

Research in power and energy optimizations focuses not only on reducing overall program energy consumption, but also on improving time-dependent power behavior. Evaluating such optimizations requires both accurate total energy consumption estimation and precise detailed time-dependent power behavior. Simulators are often used for power and performance evaluation, but detailed power simulation is very time-consuming and often inaccurate. While physical measurement is much faster, fine-grained power measurement requires proper measurement equipment and a large amount of space to store measurement results.

An example optimization that requires fine-grained, time-dependent power behavior information for its experimental evaluation is instruction scheduling for peak power 
and step power (dI/dt problem) reduction, for instance in the context of VLIW architectures $[20,19,18]$. This previous work relies on simulation to evaluate the impact of the proposed optimizations. The $\mathrm{dI} / \mathrm{dt}$ problem is caused by large variations of current in a short time. Such variations in CPU current may cause undesired oscillation in CPU supply voltage, which may results in timing problems and incorrect calculations [5]. In this paper, we introduce a new strategy to enable time-dependent power behavior characterizations based on physical measurements.

\subsection{Characterizing Phases with Representative Slices}

Program phase behavior shows that many program execution slices have similar behavior in several metrics, such as instructions-per-cycle (IPC), cache miss rate, and branch misprediction rate. Phase classification makes it easier to measure the fine-grained program behavior. A representative slice from each phase instead of the whole program execution is measured and analyzed, and then the whole program behavior can be characterized based on the analysis result. Using this whole program behavior characterization method in power behavior analysis, we can obtain fine-grained power behavior with significant savings in both time and storage space.

\subsection{Illustrating Time-Dependent Power Behavior}

Figure 1 shows the measured CPU current of 256.bzip2 from SPEC CPU 2000 measured using an oscilloscope. Figure 1(a) shows that the program execution can be roughly partitioned into 4 phases based on its power behavior. One representative slice from each phase can be measured to characterize the detailed power behavior of the benchmark. Figure 1(b) is the measured power behavior of half of a second in the first phase with a resolution that is 100 times higher than the one used for Figure 1(a). There is a repeated power behavior period of 300 milliseconds. Figure 1(c) shows the detailed power behavior of a piece of 0.05 second, from 0.1 second to 0.15 second in Figure 1(b). It shows repeated power behavior periods of less than 5 milliseconds, indicating possible finer phase classification than Figure 1(b). Also, finer measurement gives more information of time-dependent CPU power due to the resolution of the oscilloscope that we use for power measurement. The oscilloscope reports the average power for a given time granularity. This is the reason why the difference between the observed peak power (peak current) in Figure 1(a) and (c) is almost 6 Watts (0.5 amperes).

\subsection{An Infrastructure for Characterizing Time-Dependent Power Behavior}

In this paper, we present our infrastructure for program time-dependent power behavior characterization and optimization evaluation. Our Camino compiler statically instruments the assembly code of a program for profiling and physical measurement. A SimPoint-like [16] method is used for phase classification. SimPoint identifies several intervals, or simpoints, of program execution that characterize the behavior of the entire program execution. It is often used to speed up simulation by simulating only the simpoints and estimating, for instance, IPC, by taking a weighted average of the IPCs of each simpoint. 


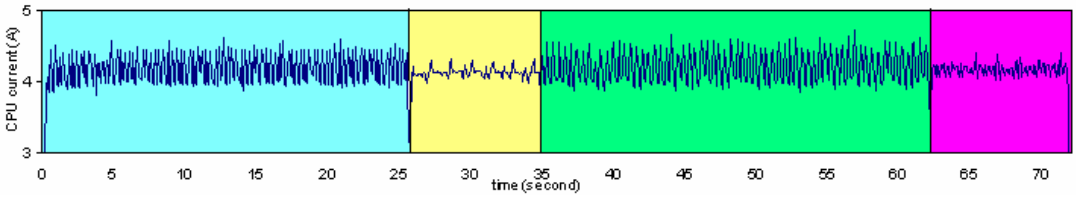

(a) Very coarse granularity

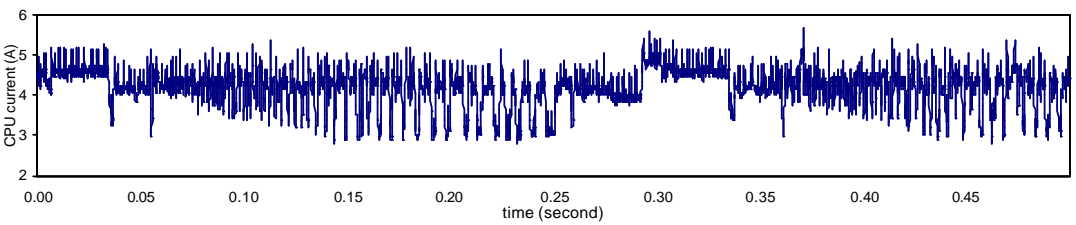

(b) A slice in phase 1 of (a)

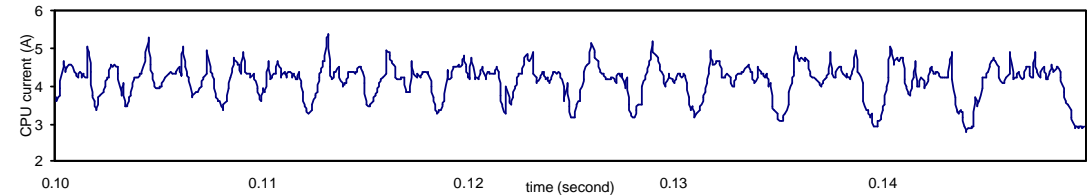

(c) Detailed CPU power behavior of a small slice from 0.10 second to 0.15 second in (b)

Fig. 1. Measured power behavior of bzip2 with different granularity.

SimPoint uses the Basic Block Vector (BBV), i.e., a vector of counts of basic block executions, as the feature for classification. We introduce the edge vector (EV), i.e., a vector of counts of control-flow-graph edge traversals, as the fingerprint of each interval of the program execution.

Instead of using a fixed number of instructions as interval length, we use infrequently executed basic blocks to demarcate intervals. This results in variable interval length, but much lower instrumentation overhead for physical power measurement of a representative interval. The selected simpoints are weighted based on the number of instructions executed in each phase, instead of number of intervals.

We show that our method enables us to do power measurement for simpoints with very low interference to program execution. To demonstrate the improved accuracy of using edge vectors for classification, we show that our infrastructure estimates the total energy of a program with an average error of $7.8 \%$, compared with $12.0 \%$ using basic block vectors, an improvement of 35\%. More importantly, we want to find representative intervals that represent the fine-grained time-dependent power profile of a phase. We develop a metric for measuring the accuracy of estimating a power profile and show that using edge vectors with event counter information improves accuracy by $22 \%$.

Unlike simulation, physical measurement is sensitive to the overhead for identification of simpoints during program execution. So this low instrumentation overhead is very important. This infrastructure can be used to evaluate optimizations for energy 
consumption or time-dependent power behavior, for example, the impact on power behavior of pipeline gating [13] or dynamic voltage/frequency scaling [6].

We evaluate our infrastructure by measuring 10 SPEC CPU2000 integer benchmarks on a Pentium 4 machine, and we present the error rates in whole program energy consumption estimation as well as fine-grained power behavior estimation based on the measurement result of the selected simpoints.

This paper makes the following contributions: 1 ) We show that using edge vectors significantly improves accuracy over using basic block vectors for estimating total program energy as well as fine-grained power behavior. 2) We show that classification accuracy can further be improved by combining control-flow information such as edge vectors with event counter information. 3) We present our infrastructure that uses edge vectors and event counters to select representative intervals and to measure efficiently their power profiles with minimal perturbation of the running program.

The rest of this paper is organized as follows. Section 2 introduces some previous work related to this research. Section 3 presents our EV-based phase classification method, its implementation using our Camino compiler, and a finer phase classification using the combination of EV and IPC. Section 4 describes our physical measurement infrastructure for precise power measurement of selected intervals. and the experiments performed to evaluate our methodology. Section 5 shows the experimental results. Section 6 concludes the paper.

\section{Related Work}

Several techniques have been proposed to identify program phases. Some of them use control-flow information $[15,16,14,11,12]$, such as counts of executed instructions, basic blocks, loops, or functions, as the fingerprint of program execution. This fingerprint depends on the executed source code. Some methods depend on run-time event counters or other metrics $[2,3,17,9]$, such as IPC, power, cache misses rate and branch misprediction, to identify phases. Our infrastructure uses the edge vector of each interval, a vector that gives a count for each control-flow edge in the program, along with the measured IPC. This set of features allows for a precise characterization of power phases.

SimPoint $[15,16]$ partitions a program execution into intervals with the same number of instructions and identifies the phases based on the BBV of each interval. One interval, called a simpoint, is selected as the representative of its phase. These simpoints are simulated or executed to estimate the behavior of the whole program execution. Sherwood et al. apply SimPoint to SPEC benchmarks to find simpoints and estimate the IPC, cache miss rate, and branch misprediction rate. The error rates are low and the simulation time saving is significant. A newer version of SimPoint supports variable length intervals [12]. That study shows a hierarchy of phase behavior in programs and the feasibility of variable length intervals in program phase classification. Procedure boundaries are used to separate variable length phases. We use infrequent basic blocks to break up intervals and at the same time use a pre-defined length to avoid intervals that are too long or too short, satisfying our requirements for low overhead and accuracy. 
Shen et al. [14] propose a data locality phase identification method for run-time data locality phase prediction. A basic block that is always executed at the beginning of a phase is identified as the marker block of this phase, resulting in variable interval lengths. They introduce the notion of a phase hierarchy to identify composite phases.

PowerScope [4] maps energy consumption to program structure through runtime system power measurement and system activity sampling. System components responsible for the bulk of energy consumption are found and improved. The delay between power sampling and activity sampling results in possible imprecise attribution of energy consumption to program structure. Compared to the power measurement granularity used by PowerScope, which is $1.6 \mathrm{~ms}$, our infrastructure measures CPU current with much higher granularity. 1000 samples are collected for each 4ms. Precise mapping between power measurement and program structure is achieved through measuring the selected representative intervals.

Isci and Martonosi [1] show that program power behavior also falls into phases. $\mathrm{Hu}$ et al. propose using SimPoint to find representative program execution slices to simplify power behavior characterization, and validate the feasibility of SimPoint in power consumption estimation through power simulation of some Mediabench benchmarks [7]. Isci and Martonosi [10] compare two techniques of phase characterization for power and demonstrate that the event-counter-based technique offers a lower average power phase classification errors. Our goal is to characterize the time-dependent power behavior, instead of power consumption, of programs. Our method causes negligible overhead for identification of an interval during program execution, and the measurement result is very close to the real time-dependent power behavior of the interval. Furthermore, through the combination of edge vector and event counters, we get better phase characterization than using only control flow information as well as the mapping between observed power behavior and the source code. The latter is difficult for an event-counter-based technique by itself.

\section{Phase Classification Based on Edge Vectors and Event Counters}

This section describes our phase classification infrastructure. It is based on the ability to demarcate the start and end of a particular interval of execution with infrequently executed basic blocks. We instrument these infrequent basic blocks so that our instrumentation minimally perturbs the execution of the program.

Phase classification and power measurement of representative intervals for programs is implemented as an automatic process. The threshold for determining whether a basic block is infrequent, the minimum number of instructions in each interval, and the number of phases are the input to this process. The flowchart in Figure 2 illustrates its steps. The implementation of each step will be presented in the following sections.

\subsection{Instrumentation Infrastructure for Profiling, Measurement, and Optimization}

Camino [8] is a GCC post-processor developed in our lab. We use it to implement the static instrumentation for profiling and physical power measurement. 

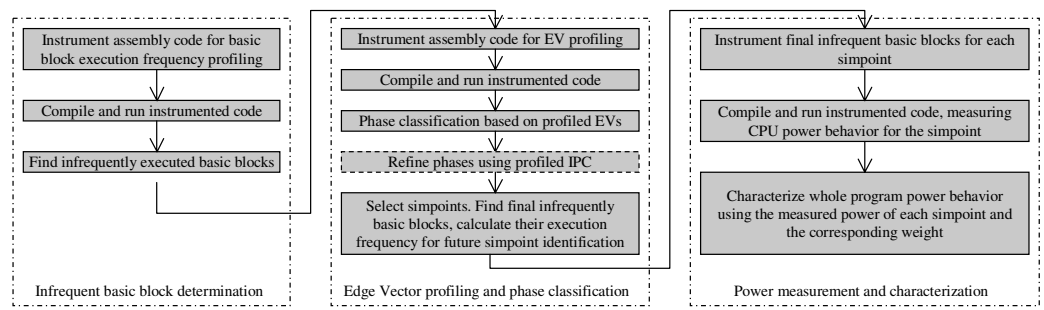

Fig. 2. Infrequent basic block-based phase classification and power measurement of simpoints.

Camino reads the assembly code generated by GCC, parses it into a control-flowgraph (CFG) intermediate representation, performs transformations including instrumentation, and then writes the modified assembly language to a file to be assembled and linked.

Instrumentation using Camino is simple and minimally intrusive. Only two routines are required: an instrumentation routine that inserts a call to the analysis routine, and and an analysis routine that does profiling or generates special signals.

Note that our infrastructure does two kinds of instrumentations: 1) profiling for all basic blocks to identify infrequent basic blocks and gathering features used to do phase classification, and 2) infrequent basic block instrumentation for signaling the start and end of a representative interval to our measurement apparatus. The first kind of instrumentation results in a moderate slowdown, but the second kind results in no slowdown so that the measured program's behavior is as close as possible to that of the uninstrumented program.

\subsection{Infrequent Basic Blocks Selection}

Instrumentation is done through Camino to collect the execution frequency of each basic block. Each basic block makes a call to an execution frequency counting library function. The distinct reference value of the basic block is passed to the function that increments the frequency of this basic block. During the first profiling pass, we collect counts for each basic block.

A threshold is needed to determine which basic blocks are infrequently executed and can be used to demarcate intervals. An absolute value is infeasible, since different program/input pairs execute different number of basic blocks. Instead, we consider a basic block to be infrequent if it account for less than a certain percentage of all executed basic blocks. Intuitively, when a low threshold is used, the selected infrequent basic blocks will be distributed sparsely in program execution and there is more variance in interval size than when a higher threshold is used. We investigate 4 different threshold values, $0.05 \%, 0.1 \%, 1 \%$, and $5 \%$, to explore the trade-off between interval size variance and instrumentation overhead. 


\subsection{Program Execution Interval Partitioning and Edge Vector Profiling}

We use the edge vector (EV) of all edges as the fingerprint of an interval used for the clustering phase of our SimPoint-like phase classification method. This vector is the absolute count for each control-flow edge traversed during the execution of an interval. Compared to basic block vectors (BBV), EVs give us more information about the control behavior of the program at run-time. BBVs contain information about what parts of a program were executed, but EVs tell us what decisions were made in arriving at these parts of the program. This extra information allows a classification of phases that more accurately reflects program behavior.

Partitioning program execution just based on the execution times of infrequent basic blocks will results in intervals with a variable number of instructions. Acquiring detailed information from very large intervals to characterize program behavior is inefficient. Moreover, a large variance in interval size affects the accuracy of the phase classification result. In order to make use of our physical measurement infrastructure to characterize the whole program behavior more correctly and efficiently, we use a pre-specified interval size to avoid largely variable intervals.

Instrumentation for EV profiling is similar to that for basic block execution frequency profiling. All basic blocks are instrumented so that we can get the complete fingerprint of an interval. An initial interval size of 30 million instructions is used to avoid too large or too small intervals. The library function remembers the last executed basic block and knows the taken edge based on the last and the current executed basic blocks. It counts each control flow edge originating in a basic block that ends in a conditional branch. It counts the total number of executed instructions for the current interval as well. When an infrequent basic block is encountered, if the count is larger than or equal to 30 million, this basic block indicates the end of the current interval and it is the first basic block of the next interval.

\subsection{Phase Classification for Power Behavior Characterization}

Intervals profiled in Section 3.3 are classified into phases based on their EVs. K-Means clustering is used to cluster the intervals with similar EVs and select a representative for each phase. The EV of each interval is projected to a vector with much smaller dimension. Then $k$ initial cluster centers are selected. The distance between a vector and each center is calculated and each vector is classified into the cluster with the shortest distance. A cluster center is changed to the average of the current cluster members after each iteration. The iteration stops after the number of vectors in each cluster is stable. The simpoint of a phase is the one that is closest to the center of the cluster [16].

Since the intervals are demarcated by infrequently executed basic blocks and have variable number of instructions, weighting a simpoint with just the number of intervals in its phase cannot reflect the real proportion of this phase in whole program execution. In our method, each simpoint has two weights. One is based on the percentage of the number of executed instructions of the corresponding phase in that of the whole program, the other is based on the number of intervals in the corresponding phase as the one used in [16]. A recent version of the SimPoint tool also supports variable-length phases [12]. 
Given the number of phases, K-Means clustering is performed for different number of clusters and different cluster seeds. The BIC (Bayesian Information Criterion) score of each clustering is calculated and used to choose the clustering with the best trade-off between BIC score and number of phases. The BIC score calculation in our method is changed to use the number of executed instructions in each phase such that phases with longer intervals have larger influence.

To identify an interval during program execution, we need to find the beginning and end of the interval. We use the execution frequencies of one or two infrequent basic blocks that demarcate the interval. Infrequent basic blocks that mark the start or end of a desired representative interval are chosen as final infrequent basic blocks. Their execution frequencies in each interval are recorded, so that we know how many times a basic block has executed before the start of an interval. We instrument these final infrequent basic blocks with simple assembly code to increment a counter and trigger power measurement when the count indicates the beginning of the interval, or turn off measurement when the end of an interval is reached. The combination of infrequent basic blocks and static instrumentation enables us to identify the execution of an interval at run-time with negligible overhead.

\subsection{Finer Phase Classification Using IPC}

Two intervals that execute the same basic blocks may generate different time-dependent power behavior due to run-time events, such as cache misses and branch mispredictions. Phase classification only based on control flow information cannot precisely differentiate these intervals, so the resulting simpoints may not really be representative in terms of power behavior. Our infrastructure combines EV and instructions-per-cycle (IPC) as measured using performance counters provided by the architecture to take the run-time events into account.

IPC Profiling Profiling IPC is easy to do in our infrastructure. After the program execution is partitioned into intervals, all of the infrequent basic blocks that demarcate the resulting intervals are instrumented to collect the number of clock cycles taken by each interval. By running the instrumented program once, we can get the IPC values of all intervals by dividing the number of instructions by the number of cycles. We already have the number of instructions executed from the edge vector profiling. Since we identify intervals based on infrequent basic block counts, the overhead is low and has a negligible impact on the accuracy of the profiling result.

Combining EV Clustering with IPC Clustering For a program execution, we first perform the phase classification in Section 3.4 to group intervals with similar EVs together. Then we do another phase classification based on the profiled IPC values. KMeans clustering is also used in the second phase classification. Then we combine the results from the two classifications and get a refined phase classification for power behavior characterization through refining the classification result of the first one using that of the second one. Suppose the number of clusters from the two classifications are $N_{e v}$ and $N_{i p c}$ respectively. The initial number of final clusters is $N_{e v} \times N_{i p c}$. Let $C_{e v}$ be 
the cluster ID of an interval in the first classification and $C_{i p c}$ be the one in the second classification. The cluster of an interval $C$ is determined by:

$$
\left.C=\left(C_{e v}-1\right) \times N_{i p c}+\left(C_{i p c}-1\right)\right)+1
$$

where $C_{e v}$ ranges from 1 to $N_{e v}$ and $C_{i p c}$ ranges from 1 to $N_{i p c}$. It is unlikely that the IPC values of every cluster in classification 1 fall into $N_{i p c}$ clusters in classification 2, so many clusters are empty. These clusters are pruned and cluster IDs are adjusted to get continuous IDs. The mechanism in the next section performs more control on the number of the resulting phases without a significant loss in accuracy. Our experiment result shows that after applying the controlling mechanism, if the given $\mathrm{K}$ value for IPC-based phase classification, that is, $C_{i p c}$ in the above equation, is 10 , the number of the resulting phases after the classification refinement is expanded to less than 3 times of the number after the first classification, instead of around 10 times.

Controlling Unnecessarily Fine Phase Classification Using a constant K value for the IPC-based phase classification of all programs results in unnecessarily fine partitioning and more simpoints to simulate or measure when the IPC values of the intervals in the same phase are already very close to each other. We control the number of resulting phases based on IPC in two steps.

The first step controls the selection of the initial centers based on the maximum and minimum IPC of the program. A percentage of the minimum IPC value is used as the distance $d$ between the initial centers. This ensures that intervals with very close IPCs need no further partitioning and the final number of simpoints does not explode with little benefit. This percentage is adjustable in our infrastructure. The maximum value is divided by $d$. The value of quotient plus 1 is then compared with the given $k$. The smaller one is used as number of clusters. This value may be 1 , meaning that the IPC values of all of the intervals are very close and no finer partitioning is necessary.

The second step maintains the distance between centers during the initialization of the centers in case there is a IPC much higher than others, but there are only two different IPC values during program execution. The first step does not know this and the number of clusters will be $k$ which results in unnecessarily more simpoints. This step is similar to the construction of a minimum spanning tree except that we use the largest values in each step to choose the next initial center. The first initial center is selected randomly. During the generation of the other initial centers, each time the value with largest distance to the existing centers is the candidate. If this distance value is less than half of $d$, no more initial centers are generated. This prevents intervals with the similar EVs and very close IPCs from being partitioned into different clusters.

\section{Experimental Setup}

We validate our infrastructure through physical power measurement of the CPU of a Pentium 4 machine. This machine runs Linux 2.6.9, GCC 3.4.2 and GCC 2.95.4. Benchmarks are from the members of SPEC CPU2000 INT that can be compiled by Camino successfully. The back-end compiler for gzip, vpr, mcf, parser and twolf is GCC 3.4.2. 
The back-end compiler for the other benchmarks is GCC 2.95 .4 because the combination of Camino and GCC 3.4.2 fails to compile these programs correctly. We measure the current on the separate power cable to the CPU using a Tektronix TCP202 DC current probe, which is connected to a Tektronix TDS3014 oscilloscope. The data acquisition machine is a Pentium 4 Linux machine that reads data from the oscilloscope when a benchmark is running on the measured system. Simultaneous benchmark execution and power data acquisition on different machines eliminates interference with the measured benchmark.

\subsection{Energy Consumption Estimation Based on Simpoints}

The first step to verify that this infrastructure is useful in power behavior characterization is to calculate the error rate when the measurement result of the selected simpoints is used to estimate the power consumption of the whole program. Although we use EVs as the fingerprint of an interval in our infrastructure, we also measured the CPU power of the simpoints using BBVs for comparison.

The energy consumption of each simpoint is measured using the trigger mode of the oscilloscope. We generate an executable for each simpoint and measure the simpoints one by one so we can get very high resolution as well as the lowest possible instrumentation overhead. Program execution and data acquisition are on the same machine. Reading data from the oscilloscope is scheduled after the measurement of a simpoint is done. Data acquisition does not interfere with the running program. We implement an automatic measurement and data acquisition process to measure any number of simpoints as a single task.

\subsection{Power Behavior Similarity Evaluation}

Even though we can get low error rates in estimating whole program energy consumption, energy consumption is the average behavior of an interval. Intervals that are classified into the same phase may have different time-dependent power behavior. If intervals in the same phase have largely different power behavior, we cannot characterize the time-dependent power behavior of the whole program execution using the measurement result of the simpoints.

Comparing in the Frequency Domain Our power measurements come in the form of discrete samples in the time domain. Power behavior is characterized by periodic activity, so a comparison in the frequency domain is more appropriate for determining whether two intervals are similar. For instance, two power curves might be slightly out of phase with one another, but have exactly the same impact on the system because they exhibit the same periodic behavior. Thus, we compare the power behavior similarity of two intervals by comparing their discrete Fourier transforms computed using Fast Fourier Transform (FFT). 
A More Robust Sampling Approach for Verification Measuring every interval in a long-running program is infeasible because of time and space constraints (indeed, this fact motivates our research). Thus, we use a more robust sampling methodology to verify that power behavior is consistent within a phase. We choose 20 intervals at random for each phase of each program to compare the FFT results of their curves. If the number of intervals in some phase is less than 20, all of the intervals are selected. The selected intervals for each phase are selected from a uniformly random distribution among all the intervals in the phase.

Instrumenting for Verification Infrequent basic blocks demarcating the intervals from the same phase are instrumented to measure each interval in the same way we measure a simpoint. Each selected interval is measured separately. Then the FFT is performed on the measured power curve of each interval. The Root Mean Square (RMS) error of the FFT results is used to evaluate the variation of the power behavior of the intervals in this phase. For each phase, we calculate the arithmetic average of the FFT results of all measured intervals as the expected FFT of the phase. The distance between an interval $i$ and the expected FFT is:

$$
D_{i}=\sqrt{\frac{\sum_{j=1}^{N}\left(\sqrt{c_{j}^{2}+d_{j}^{2}}-\sqrt{a_{j}^{2}+b_{j}^{2}}\right)}{N}}
$$

$c_{j}$ and $d_{j}$ are the real and imaginary part of the $j t h$ frequency of interval $i$, respectively. $a_{j}$ and $b_{j}$ are the real and imaginary part of the $j t h$ frequency of the expected FFT respectively. $N$ is the number of points used in Fast Fourier Transform. Then the FFT RMS of a phase is calculated as:

$$
F F T_{R M S}=\sqrt{\frac{\sum_{i=1}^{M} D_{i}^{2}}{M}}
$$

$M$ is the number of measured intervals in the phase. The lower $F F T_{R M S}$ is, the high the similarity among the time-dependent power behavior of the intervals in the phase.

The $F F T_{R M S}$ for each phase is then weighted by the weight of the corresponding phase to get the RMS for the whole benchmark. We evaluated the weighted $F F T_{R M S}$ for all of the 10 benchmarks in two cases: when phase classification is based on EV only, and when IPC is used to refine phase classification.

\section{Experimental Results and Evaluation}

Using the power measurement infrastructure described in Section 4, we measured the CPU power curves for the instrumented benchmarks, the ones with all final infrequent basic blocks instrumented, the simpoints, and the selected intervals from each phase. 


\subsection{Total Energy Consumption Estimation}

We investigate both BBV and EV as the fingerprint of intervals in phase classification. A maximum number of clusters, 30 , is used to find the best clustering in both cases. Simpoints are measured and the whole program energy consumption is estimated as

$$
E_{e s t}=\sum_{i=1}^{k} E_{i} \times W_{i}
$$

$E_{i}$ is the measured energy consumption of the $i$ th simpoint, $W_{i}$ is its weight, and $k$ is the number of phases. Although intervals have variable sizes, we estimate the total energy consumption using the weight based on the number of intervals in each phase.

For BBV-based phase classification, we use three percentage values $0.1 \%, 1 \%$, and $5 \%$ to get the threshold for infrequent basic blocks. When the measured energy consumption of simpoints are used to estimate the whole program energy consumption. The error rate is the lowest when threshold is $1 \%$ due to the trade-off between uniform interval size and instrumentation overhead. Then we use $1 \%, 0.1 \%$ and $0.05 \%$ as threshold in EV-based phase classification. Energy consumption of a measured benchmark or simpoint is calculated as:

$$
E=U \times \sum(I \times t)
$$

where $E$ is energy consumption, $U$ is the voltage of the measured CPU power cable, $I$ is the measured current on the CPU power cable, $t$ is the time resolution of the power data points. The sum is over all of the data points for one benchmark or simpoint.

Energy estimation error rate is calculated as

$$
\text { error }=\frac{\mid \text { energy_estimated }- \text { energy_measured } \mid}{\text { energy_measured }}
$$

Execution time estimation is similar to energy estimation.

Figure 3 shows the error rates of the infrequent basic block-based phase classification method using different program execution fingerprints. Error rate is calculated as in 5.1. The error reported is that of the estimate using the threshold that delivered the minimum overall error for each method: $1 \%$ for BBVs, and $0.1 \%$ for EVs. The figure shows that EV performs better than BBV for almost all of the benchmarks. EV improves the estimation accuracy by $35 \%$. All of the following analysis and evaluation are for the experimental results of EV-based phase classification.

\subsection{Time-dependent Power Behavior Similarity}

As mentioned in Section 4.2, we use the distance between the FFT results of their power curves to evaluate the similarity of two intervals in terms of power behavior. We use 4096 points in the Fast Fourier Transform. The maximum number of data points for a curve is 10,000 when the oscilloscope is in trigger mode. If the measured data points for the curve of an intervals is less than 4096, the curve is repeated to reach the number of frequencies. Figure 4 (a) shows the measured CPU current curves of two intervals 


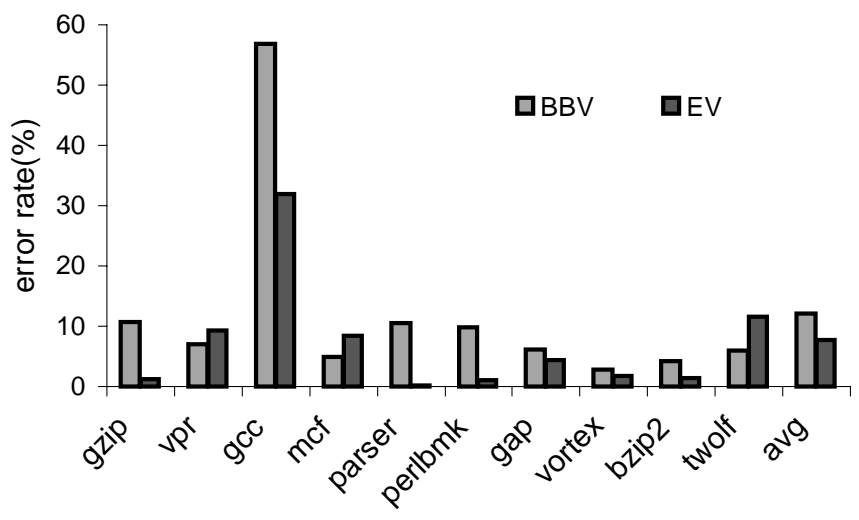

Fig. 3. Error rates of energy consumption estimation using different thresholds.

from the same identified phase, while (b) shows that of two intervals from two different phases. Distance between the FFT values is included to show the relation between time-dependent power behavior similarity and FFT distance. In Figure 4 (a), the upper curve uses the left $y$ axis, while the other one use the right $y$ axis, to avoid overlapping curves. The second column of each group in Figure 5 is the weighted $F F T_{R M S}$ for each benchmark when EV is used for phase classification.

We measure the IPC using performance counters for each interval and do phase classification based on IPC to refine the EV-based phase classification. The third column in each group in Figure 5 is the weighted $F F T_{R M S}$ for each benchmark when EV+IPC is used for phase classification. The similarity among the intervals is improved by $22 \%$ over using BBVs. Compared to the FFT distance between an interval and another interval from a different phase, the distance inside a phase is much smaller. This shows that the combination of EV and IPC enables us to classify intervals into phases in which the intervals have similar power behavior. Thus the power behavior of the whole program can be characterized by the measured behavior of the simpoints.

\section{Conclusion}

This paper introduced our infrastructure for efficient program power behavior characterization and evaluation. We presented a new phase classification method based on edge vectors combined with event counters. We described the physical measurement setup for precise power measurement. By demarcating intervals using infrequently executed basic blocks, we find intervals with variable lengths and negligible instrumentation overhead for physical measurement of simpoints. Through experiments on a real system, we demonstrated that our new phase classification method can find representative intervals for energy consumption with an accuracy superior to using basic block vectors. More importantly, we demonstrated the ability of our infrastructure to characterize the fine-grained time-dependent power behavior of each phase in the program 


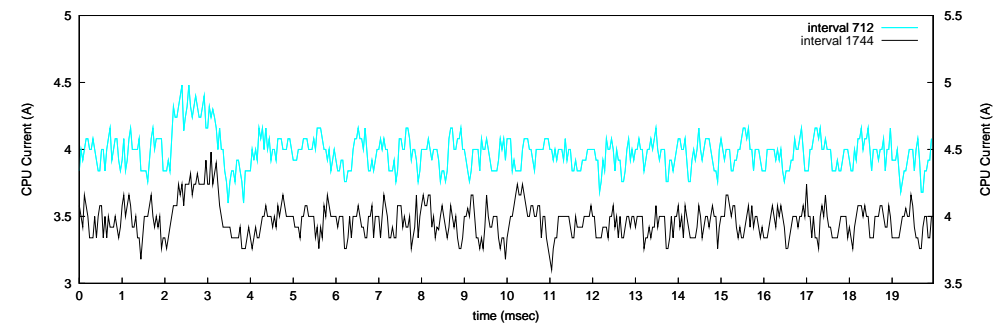

(a) Power curves of intervals from the same phase(distance=5.4).

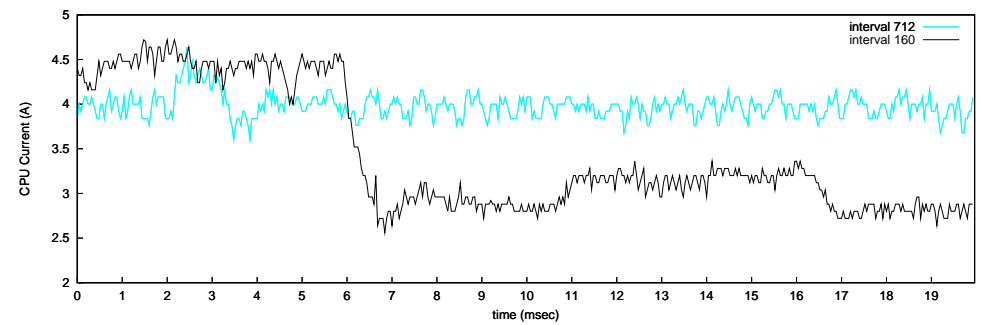

(b) Power curves of intervals from different phases(distance=55.1).

Fig. 4. Similarity between measured CPU current of intervals.

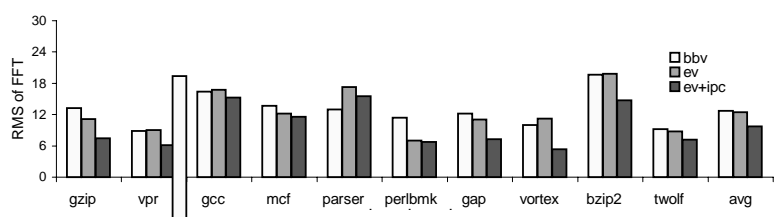

Fig. 5. Root Mean Squared error of the FFT calculated based on RMS of FFT and the weight of each phase.

using a single representative interval per phase. The ability of instrumenting programs on various levels, identifying phases, and obtaining detailed power behavior of program execution slices makes this infrastructure useful in power behavior characterization and optimization evaluation.

\section{References}

1. Canturk Isci, Margaret Martonosi. Runtime power monitoring in high-end processors: Methodology and empirical data. Proceedings of the 36th Annual IEEE/ACM International Symposium on Microarchitecture (MICRO'03), page 93, 2003.

2. E. Chi, A. M. Salem, and R. I. Bahar. Combining software and hardware monitoring for improved power and performance tuning. Proceedings of the Seventh Annual Workshop on Interaction between Compilers and Computer Architectures (INTERACT'03), 2003. 
3. E. Duesterwald, C. Cascaval, and S. Dwarkadas. Characterizing and predicting program behavior and its variability. Proceedings of the 12th International Conference on Parallel Architectures and Compilation Techniques (PACT'03), page 220, 2003.

4. J. Flinn and M. Satyanarayanan. Powerscope: A tool for profiling the energy usage of mobile applications. Proceedings of the Second IEEE Workshop on Mobile Computer Systems and Applications, page 2, 1999.

5. K. Hazelwood and D. Brooks. Eliminating voltage emergencies via microarchitectural voltage control feedback and dynamic optimization. August 2004.

6. C.-H. Hsu and U. Kremer. The design, implementation, and evaluation of a compiler algorithm for cpu energy reduction. Proceedings of the ACM SIGPLAN 2003 conference on Programming language design and implementation (PLDI'03), pages 38-48, 2003.

7. C. Hu, D. A. Jiménez, and U. Kremer. Toward an evaluation infrastructure for power and energy optimizations. In 19th International Parallel and Distributed Processing Symposium (IPDPS'05, Workshop 11), CD-ROM / Abstracts Proceedings, April 2005.

8. C. Hu, J. McCabe, D. A. Jiménez, and U. Kremer. The camino compiler infrastructure. SIGARCH Comput. Archit. News, 33(5):3-8, 2005.

9. C. Isci and M. Martonosi. Identifying program power phase behavior using power vectors. Proceedings of the IEEE International Workshop on Workload Characterization (WWC-6), 2003.

10. C. Isci and M. Martonosi. Phase characterization for power: Evaluating control-flow-based and event-counter-based techniques. In 12th International Symposium on High-Performance Computer Architecture (HPCA-12), Febrary 2006.

11. A. Iyer and D. Marculescu. Power aware microarchitecture resource scaling. Proceedings of the conference on Design, Automation and Test in Europe (DATE'01), pages 190-196, 2001.

12. J. Lau, E. Perelman, G. Hamerly, T. Sherwood, and B. Calder. Motivation for variable length intervals and hierarchical phase behavior. In the Proceedings of the IEEE International Symposium on Performance Analysis of Systems and Software (ISPASS'05), pages 135-146, 2005.

13. S. Manne, A. Klauser, and D. Grunwald. Pipeline gating: speculation control for energy reduction. Proceedings of the 25th Annual International Symposium on Computer Architecture (ISCA'98), pages 132-141, 1998.

14. X. Shen, Y. Zhong, and C. Ding. Locality phase prediction. Proceedings of the 11th International Conference on Architectural Support for Programming Languages and Operating Systems (ASPLOS'04), pages 165-176, 2004.

15. T. Sherwood, E. Perelman, and B. Calder. Basic block distribution analysis to find periodic behavior and simulation points in applications. Proceedings of the 2001 International Conference on Parallel Architectures and Compilation Techniques (PACT'01), pages 3-14, 2001.

16. T. Sherwood, E. Perelman, G. Hamerly, and B. Calder. Automatically characterizing large scale program behavior. In Proceedings of the 10th International Conference on Architectural Support for Programming Languages and Operating Systems (ASPLOS'02), October 2002.

17. R. Srinivasan, J. Cook, and S. Cooper. Fast, accurate microarchitecture simulation using statistical phase detection. Proceedings of The 2005 IEEE International Symposium on Performance Analysis of Systems and Software (ISPASS'05), 2005.

18. C.-L. Su, C.-Y. Tsui, and A. Despain. Low power architecture and compilation techniques for high-performance processors. In IEEE COMPCON, pages 489-498, San Francisco, CA, February 1994.

19. M. Toburen, T. Conte, and M. Reilly. Instruction scheduling for low power dissipation in high performance microprocessors. In Power Driven Microarchitecture Workshop, Barcelona, Spain, June 1998.

20. H.-S. Yun and J. Kim. Power-aware modulo scheduling for high-performance VLIW. In International Symposium on Low Power Electronics and Design (ISLPED'01), Huntington Beach, CA, August 2001. 